\title{
An Investigation of Post-radiation Gene Expression Profiles: A System Biology Study
}

\author{
Reza Vafaee $^{1}{ }^{\circledR}$, Abdolrahim Nikzamir ${ }^{2}$, Mohhamadreza Razzaghi ${ }^{3}$, Sina Rezaei Tavirani ${ }^{4}$, Alireza \\ Ahmadzadeh $^{2}$, MohammadAli Emamhadi ${ }^{*}$ (D) \\ 'Proteomics Research Center, Faculty of Paramedical Sciences, Shahid Beheshti University of Medical Sciences, Tehran, \\ Iran \\ ${ }^{2}$ Faculty of Medicine, Shahid Beheshti University of Medical Sciences, Tehran, Iran \\ ${ }^{3}$ Laser Application in Medical Sciences Research Center, Shahid Beheshti University of Medical Sciences, Tehran, Iran \\ ${ }^{4}$ Gastroenterology and Liver Diseases Research Center, Research Institute for Gastroenterology and Liver Diseases, Shahid \\ Beheshti University of Medical Sciences, Tehran, Iran \\ ${ }^{5}$ Forensic Medicine Specialist, Forensic Medicine Department, Shahid Beheshti Medical University, Tehran, Iran
}

\section{*Correspondence to MohammadAli Emamhadi, Forensic Medicine Specialist, Forensic Medicine Department Shahid Beheshti Medical University, Tehran, Iran Email: emamhadi2000@gmail. com}

Published online December 30 2020

\begin{abstract}
Introduction: Genomics and bioinformatics are useful methods for exploring unclear aspects of radiation effects on biological systems. Many radiation-induced alterations in irradiated samples are post-radiation time-dependent. This study aims to evaluate the post-irradiation effects of the gamma ray on human Jurkat cells.

Methods: Gene expression profiles of the samples harvested 6 and 24 hours after radiation to find the critical differential expressed genes and the related pathways. Samples are provided from Gene Expression Omnibus (GEO) and analyzed by ClueGO.

Results: Twnety-nine critical genes were determined as the important affected genes and 7 classes of related pathways were introduced. CCNE2, PSMD11, CDC25C, ANAPC1, PLK1, AURKA, and CCNB1 that were associated with more than 6 pathways were related to one of the determined pathway groups.

Conclusion: Cell protecting pathways were associated with the genes (HSPA5, HSPA8, HSP90B1, HMMR, CEBPB, RXRA, and PSMD11) which were related to the minimum numbers of pathways. The finding of this study corresponds to repair processes which depend on post-radiation time. It seems these sets of genes are suitable candidates for further investigation.

Keywords: Radiation; Bioinformatics; Gene expression; Pathway; Dysregulation
\end{abstract}

\section{Introduction}

It is reported that gamma radiation induces chromosomal aberration and DNA damages in the exposed samples. ${ }^{1,2}$ Genomics as a high-throughput method is applied to discover the widespread aspect of gamma irradiation effects on biological systems. ${ }^{3,4}$ Since the bioinformatics approach is tied to the high-throughput methods, bioinformatics plays a significant role in the interpretation of results of gamma irradiation effects on living organisms. ${ }^{5}$

Genomics as a method which studies the genome is applied to investigate the dysregulated genes after inducing alterations in internal or external conditions of living samples. In such a study, the gene expression profiles of samples are assessed to find the targeted genes. Finally, different types of dysregulated genes with different amounts of dysregulation are introduced. ${ }^{6,7}$ Like the other large scale methods, large numbers of dysregulated genes are determined in a genomics experiment. The study and evaluation of this gene set need powerful techniques such as bioinformatics and its various branches. ${ }^{8-10}$

The products of the genes play roles in the biochemical pathways to maintain life hemostasis and health condition. Alteration in the gene expression level leads to functional changes in the related pathways, which is reflected in the lifestyle of the treated sample. ${ }^{11,12}$ There are several databases including different biochemical pathways such as the KEGG database that is applied to analyze the dysregulated pathways by many researchers. ${ }^{13,14}$ RezaeiTavirani et al have introduced "Cytokine-Mediated Signaling Pathway" as distinctive dysregulated biological terms after skin laser therapy. ${ }^{15}$ Since the DNA repair process after irradiation is an important parameter and needs proper time to decrease damages, ${ }^{16}$ in the present study, the gene expression profiles of irradiated cells by gamma rays in 6 hours and 24 hours of "post-radiation time" are studied to find the critical dysregulated genes and biochemical pathways. 


\section{Materials and Methods}

The gene profiles of GSM2792818-20 from GSE104222/

GPL6480 in GEO, which were irradiated with 10 Gy $\gamma$-ray and harvested 6 hours after radiation, were selected as controls. The gene expression profiles of the samples after 24 hours of radiation were assigned to compare.

The feasibility of the comparison of samples was provided by the assessment of the studied gene expression profiles via boxplot analysis by the GEO2R program.

Among 250 differential expressed genes (DEGs) , 190 characterized individuals were selected to be analyzed. A $P$ value $<0.001$ and a fold change $>1.5$ were considered statistically significant. If there was more than one isomer, the individual with the highest value of expression was selected. Therefore, 177 DEGs were selected to be analyzed in the next steps.

The related pathways for the 177 query DEGs were investigated in Wiki Pathways, KEGG, and REACTOME Pathways via ClueGO. The network specificity medium and $P$ value $<0.05$ were considered to find the related pathways. The pathways considering; term $P$ value, term $P$ value corrected with Bonferroni step down, group $P$ value, and group $P$ value corrected with Bonferroni step down were less than 0.05 . Kappa score $=7$ was resulted.

\section{Results}

As it is shown in Figure 1, seven classes of pathways including 31 pathways were related to 29 DEGs among the 177 query genes. The introduced pathways and the related genes are shown in Table 1. As it is depicted in Table 1, about $16 \%$ of genes among 177 query DEGs are related to the identified pathways. The percentage of the associated genes for each pathway is presented in this table. The maximum percentage of associated genes (18.75\%) is attributed to (Polo-like kinase mediated events). Based on the presence of an individual gene in several pathways, the frequency of the associated pathways for the query DEGs is calculated and presented in Table 2. CCNB1 is related to maximum pathways, while $11 \mathrm{DEGs}$ are related to one pathway. Since the identified pathways are classified in the 7 groups of pathways, the associated pathway groups for the involved query DEG are determined and shown in Table 2. As shown in Table 2, there are several genes that are common between the introduced pathway groups. For better understanding, these genes and the related pathway groups are presented schematically in Figure 2. The pathways are split into 3 sets; individuals that are linked to 3 genes including groups 5 and 6, groups 1, 3, and 7 that are connected to two genes, and finally, pathway groups of 2 and 4 which are associated with one gene.

\section{Discussion}

Biological effects of gamma-ray radiation and human health care have been investigated for many years and useful information has been obtained but there are many aspects about the mechanism of resulted damages and also protective methods that are less clear by now. ${ }^{17-}$ 19 In the present study, the effect of post-radiation time on the gene expression profile of the exposure cells was investigated. Like other high-throughput studies, ${ }^{20,21}$ gamma radiation widely changed the gene expression profile of the irradiated cells so many different types of genes were dysregulated. Bioinformatics as a useful method is applied to resolve problems which are tied to the complex data of high-throughput investigations. ${ }^{22,23}$ Thus, among the large numbers of dysregulated genes 177 DEGs were candidates to be assessed. Pathway analysis is applied to screen and also elucidate the results of such experiments, and findings are published to introduce the limited numbers of important dysregulated genes and critical affected pathways. ${ }^{24-26}$ In Figure 1, various types of pathways which are related to the dysregulated genes are presented. Further investigation is needed to explain the details of Figure 2. As shown in Table 1, the determined

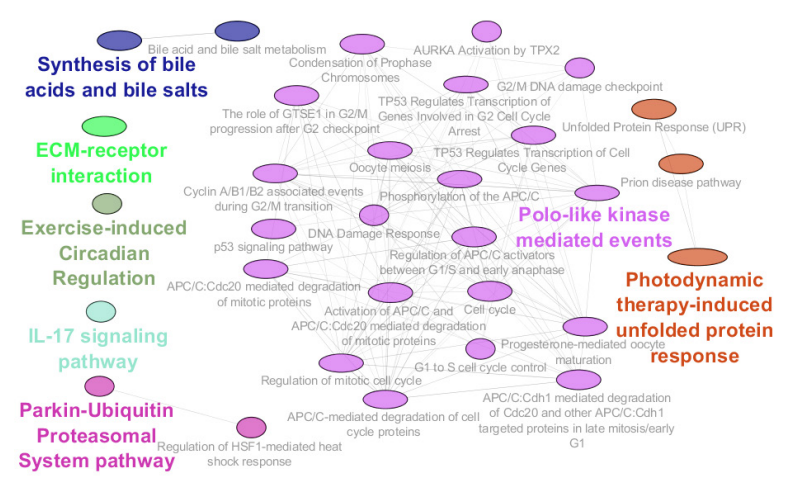

Figure 1. Results of Pathway Analysis for the 177 Query DEGs. Each color refers to an individual class of pathways.

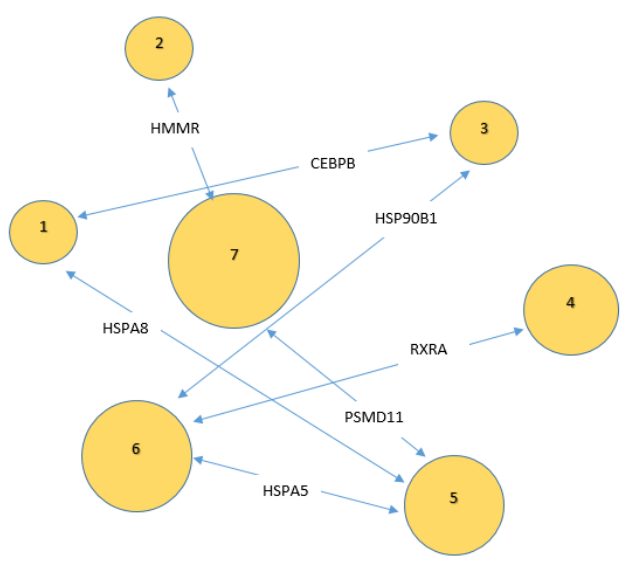

Figure 2. The genes that are common between the introduced pathway groups and the related groups. Group size is proportional to the number of pathways which are included in the group. The groups of 5 and 6 are connected to 3 genes, while groups of 1, 3, and 7 are related to two genes and groups of 2 and 4 were linked by one DEG. 
Table 1. Related Pathways for the 177 Query DEGs

\begin{tabular}{|c|c|c|}
\hline Pathway & $\%$ AG & Associated Genes Found \\
\hline${ }^{1}$ Exercise-induced circadian regulation & 6.25 & [CEBPB, DNAJA1, HSPA8] \\
\hline${ }^{2} \mathrm{ECM}$-receptor interaction & 4.88 & {$[\mathrm{HMMR}, \mathrm{SV} 2 \mathrm{~B}, \mathrm{TNN}, \mathrm{VWF}]$} \\
\hline${ }^{3}$ IL-17 signaling pathway & 4.30 & [CEBPB, HSP90B1, MAPK15, SRSF1] \\
\hline${ }^{4}$ Synthesis of bile acids and bile salts & 8.82 & [OSBPL1A, OSBPL6, RXRA] \\
\hline${ }^{4}$ Bile acid and bile salt metabolism & 6.98 & [OSBPL1A, OSBPL6, RXRA] \\
\hline${ }^{5}$ Regulation of HSF1-mediated heat shock response & 4.41 & [HSPA5, HSPA8, HSPH1] \\
\hline${ }^{5}$ Parkin-Ubiquitin Proteasomal System pathway & 4.29 & [HSPA5, HSPA8, PSMD11] \\
\hline${ }^{6}$ Unfolded protein response (UPR) & 4.08 & [ATF3, CREBRF, HSP90B1, HSPA5] \\
\hline${ }^{6}$ Photodynamic therapy-induced unfolded protein response & 14.81 & [ATF3, HSP90B1, HSPA5, TRIB3] \\
\hline${ }^{6}$ Prion disease pathway & 9.09 & [HSP90B1, HSPA5, RXRA] \\
\hline${ }^{7}$ Cell cycle & 4.03 & [ANAPC1, CCNB1, CCNE2, CDC25C, PLK1] \\
\hline${ }^{7}$ Oocyte meiosis & 4.84 & [ANAPC1, AURKA, CCNB1, CCNE2, CDC25C, PLK1] \\
\hline${ }^{7}$ p53 signaling pathway & 5.88 & [CCNB1, CCNE2, GTSE1, SESN2] \\
\hline${ }^{7}$ Progesterone-mediated oocyte maturation & 5.05 & [ANAPC1, AURKA, CCNB1, CDC25C, PLK1] \\
\hline${ }^{7}$ Polo-like kinase mediated events & 18.75 & [CCNB1, CDC25C, PLK1] \\
\hline${ }^{7} \mathrm{APC} / \mathrm{C}$-mediated degradation of cell cycle proteins & 5.81 & [ANAPC1, AURKA, CCNB1, PLK1, PSMD11] \\
\hline $\begin{array}{l}{ }^{7} \mathrm{APC} / \mathrm{C}: \mathrm{Cdh} 1 \text { mediated degradation of } \mathrm{Cdc} 20 \text { and other APC/C:Cdh1 targeted proteins } \\
\text { in late mitosis/early G1 }\end{array}$ & 5.56 & [ANAPC1, AURKA, PLK1, PSMD11] \\
\hline${ }^{7}$ Regulation of APC/C activators between G1/S and early anaphase & 5.00 & [ANAPC1, CCNB1, PLK1, PSMD11] \\
\hline${ }^{7} \mathrm{APC} / \mathrm{C}: \mathrm{Cdc} 20$ mediated degradation of mitotic proteins & 4.00 & [ANAPC1, CCNB1, PSMD11] \\
\hline${ }^{7}$ Phosphorylation of the APC/C & 15.79 & [ANAPC1, CCNB1, PLK1] \\
\hline${ }^{7}$ Activation of $\mathrm{APC} / \mathrm{C}$ and $\mathrm{APC} / \mathrm{C}: \mathrm{Cdc} 20$ mediated degradation of mitotic proteins & 5.26 & [ANAPC1, CCNB1, PLK1, PSMD11] \\
\hline${ }^{7}$ Condensation of Prophase Chromosomes & 4.05 & [CCNB1, HIST1H2BD, PLK1] \\
\hline${ }^{7}$ Regulation of mitotic cell cycle & 5.81 & [ANAPC1, AURKA, CCNB1, PLK1, PSMD11] \\
\hline${ }^{7}$ TP53 Regulates Transcription of Cell Cycle Genes & 8.16 & [AURKA, CCNB1, CCNE2, CDC25C] \\
\hline${ }^{7}$ TP53 Regulates Transcription of Genes Involved in G2 Cell Cycle Arrest & 16.67 & [AURKA, CCNB1, CDC25C] \\
\hline${ }^{7} \mathrm{C} y c$ lin $\mathrm{A} / \mathrm{B} 1 / \mathrm{B} 2$ associated events during $\mathrm{G} 2 / \mathrm{M}$ transition & 12.00 & [CCNB1, CDC25C, PLK1] \\
\hline${ }^{7} \mathrm{G} 2 / \mathrm{M}$ DNA damage checkpoint & 4.21 & [CCNB1, CDC25C, HIST1H2BD, RNF168] \\
\hline${ }^{7}$ The role of GTSE1 in G2/M progression after G2 checkpoint & 5.13 & [CCNB1, GTSE1, PLK1, PSMD11] \\
\hline${ }^{7}$ AURKA Activation by TPX2 & 4.11 & [AURKA, HMMR, PLK1] \\
\hline${ }^{7} \mathrm{G} 1$ to $\mathrm{S}$ cell cycle control & 4.41 & [CCNB1, CCNE2, PRIM1] \\
\hline${ }^{7} \mathrm{DNA}$ damage response & 4.41 & [CCNB1, CCNE2, CDC25C] \\
\hline
\end{tabular}

Note: Pathways are extracted from Wiki Pathways, KEGG, and REACTOME Pathways. Term $P$ value, term $P$ value corrected with Bonferroni step down, group $P$ value, and group $P$ value corrected with Bonferroni step-down were less than 0.05 . \%AG refers to \%associated genes. The superscript numbers in column 1 is an indicator of group number.

pathways are classified into 7 pathway groups. The groups are different based on the kind of included pathways and also the number of pathways. "Polo-like kinase mediated events" is the main group which includes 21 pathways (about $72 \%$ of total pathways), while there are three groups (groups 1-3) that include only one pathway.

As it is depicted in Table 2, only 16\% (29 DEGs) of the query genes are involved in the introduced pathways. Based on the attribution of DEGs in the pathways, the genes can be categorized as the following groups: first, based on the involvement of genes in the pathway groups, the genes that are presented merely in one group and the DEGs which are common between more than one groups; second, based on the number of related pathways for the genes, the genes with a high value of associated pathways and the genes with few relevant pathways.

As shown in Table 2, 11 genes (about 38\% of the genes) are related to one pathway while 7 individuals are related to 2 pathways. There are 3 related pathways for two genes and also 4 pathways are attributed to 2 DEGs. The genes that are involved in more than 6 pathways including CCNE2, PSMD11, CDC25C, ANAPC1, PLK1, AURKA, and CCNB1 are the genes that were associated merely with class 7 of the pathways (Polo-like kinase mediated events).

It can be concluded that the main group of pathways is "Polo-like kinase mediated events" that includes $72 \%$ of pathways and the genes with a higher degree of 
Table 2. Frequency of Associated Pathways and Pathway Groups for Certain DEGs

\begin{tabular}{|c|c|c|c|c|}
\hline $\mathbf{R}$ & Gene & Description & NAT & AGN \\
\hline 1 & CEBPB & CCAAT/enhancer binding protein beta & 2 & 1,3 \\
\hline 2 & DNAJA1 & DnaJ heat shock protein family (Hsp40) member A1 & 1 & 1 \\
\hline 3 & HSPH1 & heat shock protein family H (Hsp110) member 1 & 1 & 5 \\
\hline 4 & HSPA5 & heat shock protein family A (Hsp70) member 5 & 4 & 5,6 \\
\hline 5 & HSPA8 & heat shock protein family $\mathrm{A}(\mathrm{Hsp} 70)$ member 8 & 3 & 1,5 \\
\hline 6 & HSP90B1 & heat shock protein 90 beta family member 1 & 4 & 3,6 \\
\hline 7 & HMMR & hyaluronan mediated motility receptor & 2 & 7,2 \\
\hline 8 & SV2B & synaptic vesicle glycoprotein 2B & 1 & 2 \\
\hline 9 & TNN & tenascin $\mathrm{N}$ & 1 & 2 \\
\hline 10 & VWF & von Willebrand factor & 1 & 2 \\
\hline 11 & MAPK15 & mitogen-activated protein kinase 15 & 1 & 3 \\
\hline 12 & SRSF1 & serine and arginine rich splicing factor 1 & 1 & 3 \\
\hline 13 & OSBPL1A & oxysterol binding protein like 1A & 2 & 4 \\
\hline 14 & OSBPL6 & oxysterol binding protein like 6 & 2 & 4 \\
\hline 15 & RXRA & retinoid $\mathrm{X}$ receptor alpha & 3 & 4,6 \\
\hline 16 & PSMD11 & proteasome $26 \mathrm{~S}$ subunit, non-ATPase 11 & 8 & 5,7 \\
\hline 17 & ATF3 & activating transcription factor 3 & 2 & 6 \\
\hline 18 & CREBRF & CREB3 regulatory factor & 1 & 6 \\
\hline 19 & ANAPC1 & anaphase promoting complex subunit 1 & 10 & 7 \\
\hline 20 & CCNB1 & cyclin B1 & 19 & 7 \\
\hline 21 & CCNE2 & cyclin E2 & 6 & 7 \\
\hline 22 & CDC25C & cell division cycle $25 \mathrm{C}$ & 9 & 7 \\
\hline 23 & PLK1 & polo like kinase 1 & 14 & 7 \\
\hline 24 & AURKA & aurora kinase $\mathrm{A}$ & 8 & 7 \\
\hline 25 & GTSE1 & G2 and S-phase expressed 1 & 2 & 7 \\
\hline 26 & SESN2 & Sestrin 2 & 1 & 7 \\
\hline 27 & HIST1H2BD & histone cluster $1, \mathrm{H} 2 \mathrm{bd}$ & 2 & 7 \\
\hline 28 & PRIM1 & primase (DNA) subunit 1 & 1 & 7 \\
\hline 29 & RNF168 & ring finger protein 168 & 1 & 7 \\
\hline
\end{tabular}

Note: NAT and AGN refer to "number of the associated term" and "associated group number" respectively.

participation in the pathway are related to this pathway class. The significant roles of cyclins such as CCNE2 and CCNB1 in the regulation of the cell cycle are highlighted in the many investigations. ${ }^{27-29}$ Roles of PSMD11, CDC25C, ANAPC1, and PLK1 in the cell cycle and response to DNA damages are highlighted by Torres-Ávila et al. ${ }^{30}$ Medina-Aguilar et al published a document about the role of AURKA (Aurora kinase A) in the regulation of the cell cycle. ${ }^{31}$ It seems that pathway group 7 is tied to the regulation of the cell cycle and cell proliferation. This finding corresponds with the nature of gamma exposure.

Considering Figure 2, seven important genes are introduced as a linker between the 7 pathway groups. HSPA5, HSPA8, HSP90B1, HMMR, CEBPB, RXRA, and PSMD11 are the genes that connect all the pathway groups. It can be concluded that all groups are connected directly or indirectly to each other. HSPA5, HSPA8, HSP90B1, RXRA, and PSMD11 are down-regulated while HMMR and CEBPB are up-regulated (the data are not shown). HMMR links groups 2 (ECM-receptor interaction) and 7. The up-regulation of genes which are involved in "ECMreceptor interaction" in adipocytes that are active mainly in energy storage is confirmed by Hyun-Jeong Lee et al. ${ }^{32}$ It seems the activation of this pathway is a protective response of cells against gamma exposure. "Exerciseinduced Circadian Regulation" and "IL-17 signaling pathway" are connected by CEBPB. The up-regulation of CEBPB and the promotion of related functions increase the roles of these pathways in the protection of cells in the irradiated cells.

\section{Conclusion}

In conclusion, 29 critical genes were introduced as the dysregulated individuals by gamma-ray irradiation that is related to the lacks in the pathways which are mainly involved in cell proliferation and activation of protective 
pathways. It seems that the activation of protective pathways is supported in the cells with longer postradiation time.

\section{Ethical Considerations}

Not applicable.

\section{Conflict of Interests}

The authors declare no conflict of interest.

\section{Acknowledgments}

Shahid Beheshti University of Medical Sciences supported this research.

\section{References}

1. Kumar DS, Chakrabarty D, Verma AK, Banerji BK. Gamma ray induced chromosomal aberrations and enzyme related defense mechanism in Allium cepa L. Caryologia G Citol Citosistematica Citogenet. 2011;64(4):388-97.

2. Teoule R. Radiation-induced DNA damage and its repair. Int J Radiat Biol Relat Stud Phys Chem Med. 1987;51(4):57389. doi: 10.1080/09553008414552111.

3. Kadhim MA, Moore SR, Goodwin EH. Interrelationships amongst radiation-induced genomic instability, bystander effects, and the adaptive response. Mutat Res. 2004;568(1):21-32. doi: 10.1016/j.mrfmmm.2004.06.043.

4. Matsui A, Kobayashi J, Kanno SI, Hashiguchi K, Miyaji M, Yoshikawa $\mathrm{Y}$, et al. Oxidation resistance 1 prevents genome instability through maintenance of G2/M arrest in gammaray-irradiated cells. J Radiat Res. 2020;61(1):1-13. doi: 10.1093/jrr/rrz080.

5. Georgakilas AG, Pavlopoulou A, Louka M, Nikitaki Z, Vorgias CE, Bagos PG, et al. Emerging molecular networks common in ionizing radiation, immune and inflammatory responses by employing bioinformatics approaches. Cancer Lett. 2015;368(2):164-72. doi: 10.1016/j.canlet.2015.03.021.

6. Taylor J, Mi X, North K, Binder M, Penson A, Lasho T, et al. Single-cell genomics reveals the genetic and molecular bases for escape from mutational epistasis in myeloid neoplasms. Blood. 2020;136(13):1477-86. doi: 10.1182/ blood.2020006868.

7. St-Cyr S, Aubin-Horth N. Integrative and genomics approaches to uncover the mechanistic bases of fish behavior and its diversity. Comp Biochem Physiol A Mol Integr Physiol. 2009;152(1):9-21. doi: 10.1016/j. cbpa.2008.09.003.

8. Safaei A, Rezaei-Tavirani M, Sobhi S, Akbari ME. Breast cancer biomarker discovery: Proteomics and genomics approaches. Iran J Cancer Prev. 2013;6(Suppl):45-53.

9. Zamanian Azodi M, Rezaei-Tavirani M, Rostami-Nejad M, Rezaei-Tavirani M. Comparative bioinformatics characteristic of bladder cancer stage 2 from stage 4 expression profile: a network-based study. Galen. 2018;7:e1279. doi: 10.22086/gmj.v0i0.1279.

10. Robinson JT, Thorvaldsdóttir H, Winckler W, Guttman M, Lander ES, Getz G, et al. Integrative genomics viewer. Nat Biotechnol. 2011;29(1):24-6. doi: 10.1038/nbt.1754.

11. Kobayashi Y, Murata M, Minami H, Yamamoto S, Kagaya Y, Hobo T, et al. Abscisic acid-activated SNRK2 protein kinases function in the gene-regulation pathway of $\mathrm{ABA}$ signal transduction by phosphorylating $\mathrm{ABA}$ response element-binding factors. Plant J. 2005;44(6):939-49. doi: 10.1111/j.1365-313X.2005.02583.x.

12. Peukert K, Staller P, Schneider A, Carmichael G, Hänel F, Eilers M. An alternative pathway for gene regulation by Myc. EMBO J. 1997;16(18):5672-86. doi: 10.1093/ emboj/16.18.5672.

13. Kanehisa M. The KEGG database. Novartis Found Symp. 2002;247:91-101.

14. Kanehisa M, Furumichi M, Sato Y, Ishiguro-Watanabe M, Tanabe M. KEGG: integrating viruses and cellular organisms. Nucleic Acids Res. 2020. doi: 10.1093/nar/ gkaa970.

15. Rezaei-Tavirani M, Tavirani MR, Zamanian Azodi M, Moravvej Farshi H, Razzaghi M. Evaluation of skin response after Erbium: Yttrium-Aluminum-Garnet laser irradiation: a network analysis approach. J Lasers Med Sci. 2019;10(3):194-99. doi: 10.15171/jlms.2019.31.

16. Banáth JP, MacPhail SH, Olive PL. Radiation sensitivity, $\mathrm{H} 2 \mathrm{AX}$ phosphorylation, and kinetics of repair of DNA strand breaks in irradiated cervical cancer cell lines. Cancer Res. 2004;64(19):7144-9. doi: 10.1158/0008-5472.CAN-041433.

17. Graupner A, Eide DM, Instanes C, Andersen JM, Brede DA, Dertinger SD, et al. Gamma radiation at a human relevant low dose rate is genotoxic in mice. Sci Rep. 2016;6:32977. doi: $10.1038 /$ srep32977.

18. Gandhi NM, Nair CK. Protection of DNA and membrane from gamma radiation induced damage by gallic acid. $\mathrm{Mol}$ Cell Biochem. 2005;278(1-2):111-7. doi: 10.1007/s11010005-6940-1.

19. Aygün B, Alaylar B, Turhan K, Şakar E, Karadayı M, AlSayyed MIA, et al. Investigation of neutron and gamma radiation protective characteristics of synthesized quinoline derivatives. Int J Radiat Biol. 2020;96(11):142334. doi: 10.1080/09553002.2020.1811421.

20. Carulli JP, Artinger M, Swain PM, Root CD, Chee L, Tulig C, et al. High throughput analysis of differential gene expression. J Cell Biochem. 1998;72(S30-31):286-96. doi: 10.1002/(SICI) 1097-4644(1998)72:30/31+<286::AIDJCB35>3.0.CO;2-D.

21. Kim HK, Lee S, Kim Y, Park J, Min S, Choi JW, et al. Highthroughput analysis of the activities of xCas9, SpCas9-NG and $\mathrm{SpCas} 9$ at matched and mismatched target sequences in human cells. Nat Biomed Eng. 2020;4(1):111-24. doi: 10.1038/s41551-019-0505-1.

22. Heidari $\mathrm{MH}$, Razzaghi $M$, Akbarzadeh Baghban A, Rostami-Nejad M, Rezaei-Tavirani M, Zamanian Azodi M, et al. Assessment of the microbiome role in skin protection against UV irradiation via network analysis. J Lasers Med Sci. 2020;11(3):238-42. doi: 10.34172/jlms.2020.40.

23. Amiri-Dashatan N, Koushki M, Jalilian A, Ahmadi NA, Rezaei-Tavirani M. Integrated bioinformatics analysis of mRNAs and miRNAs identified potential biomarkers of oral squamous cell carcinoma. Asian Pac J Cancer Prev. 2020;21(6):1841-8. doi: 10.31557/APJCP.2020.21.6.1841.

24. Rezaei Tavirani M, Rezaei Tavirani S, Rostami FT. Biochemical pathway analysis of gastric atrophy. Gastroenterol Hepatol Bed Bench. 2018;11(2):118-24. 
25. Rostami-Nejad M, Rezaei-Tavirani M, Zadeh-Esmaeel MM, RezaeiTavirani S, Akbari Z, Esmaeili S, et al. Assessment of cytokine-mediated signaling pathway dysregulation in arm skin after CO2 laser therapy. J Lasers Med Sci. 2019;10(4):257-63. doi: 10.15171/jlms.2019.42.

26. Purdue MP, Song L, Scélo G, Houlston RS, Wu X, Sakoda $\mathrm{LC}$, et al. Pathway analysis of renal cell carcinoma genomewide association studies identifies novel associations. Cancer Epidemiol Biomarkers Prev. 2020;29(10):2065-9. doi: 10.1158/1055-9965.EPI-20-0472.

27. Johnson D, Walker C. Cyclins and cell cycle checkpoints. Annu Rev Pharmacol Toxicol. 1999;39:295-312.

28. Murray AW. Recycling the cell cycle: cyclins revisited. Cell. 2004;116(2):221-34. doi: 10.1016/s0092-8674(03)01080-8.

29. Ding L, Cao J, Lin W, Chen H, Xiong X, Ao H, et al. The roles of cyclin-dependent kinases in cell-cycle progression and therapeutic strategies in human breast cancer. Int $\mathrm{J} \mathrm{Mol}$ Sci. 2020;21(6):1960. doi: 10.3390/ijms21061960.
30. Torres-Ávila JF, Espitia-Pérez L, Bonatto D, da Silva FR, de Oliveira IM, Silva LF, et al. Systems chemo-biology analysis of DNA damage response and cell cycle effects induced by coal exposure. Genet Mol Biol. 2020;43(3):e20190134. doi: 10.1590/1678-4685-GMB-2019-0134.

31. Medina-Aguilar R, Marchat LA, Arechaga Ocampo E, Gariglio P, García Mena J, Villegas Sepúlveda N, et al. Resveratrol inhibits cell cycle progression by targeting Aurora kinase A and Polo-like kinase 1 in breast cancer cells. Oncol Rep. 2016;35(6):3696-704. doi: 10.3892/ or.2016.4728.

32. Lee HJ, Jang M, Kim H, Kwak W, Park W, Hwang JY, et al. Comparative transcriptome analysis of adipose tissues reveals that ECM-receptor interaction is involved in the depot-specific adipogenesis in cattle. PLoS One. 2013;8(6):e66267. doi: 10.1371/journal.pone.0066267. 